Mens

revue d'histoire intellectuelle de l'Amérique française

\title{
IV. Les idées, la ville et l'usine (1880-1929)
}

Volume 6, numéro supplément, 2005-2006

URI : https://id.erudit.org/iderudit/1024239ar

DOI : https://doi.org/10.7202/1024239ar

Aller au sommaire du numéro

Éditeur(s)

Centre de recherche en civilisation canadienne-française

ISSN

1492-8647 (imprimé)

1927-9299 (numérique)

Découvrir la revue

Citer ce document

(2005). IV. Les idées, la ville et l'usine (1880-1929). Mens, 6, 41-63.

https://doi.org/10.7202/1024239ar d'utilisation que vous pouvez consulter en ligne.

https://apropos.erudit.org/fr/usagers/politique-dutilisation/ 
SICO'T"TE, Hélène, « Le rôle de la vente publique dans l'essor du commerce d'art à Montréal au XIXe siècle - Le cas de W. Scott \& Sons ou comment le marchand d'art supplanta l'encanteur ", The Journal of Canadian Art History/Annales d'histoire de l'art canadien, 23, 1-2 (2002), p. 6-33.

SKIDMORE, Colleen, "Triumphal Arches at Montreal, 1860 ", Journal of Canadian Art History/Annales d'histoire de l'art canadien, 19, 1 (1998), p. 86-109.

SKIDMORE, Colleen, "Photography in the Convent: Grey Nuns, Quebec, 1861 », Social History/Histoire sociale, 35, 70 (2002), p. $279-310$.

THÉRIO, Adrien, Joseph Guibord : victime expiatoire de l'érêque Bourget. L'Institut canadien et l'affaire Guibord reirisités, Montréal, XYZ, 2000, $270 \mathrm{p}$.

TOCQUEVILLE, Alexis de, Regards sur le Bas-Canada, Choix de textes et présentation de Claude Corbo, Montréal, Typo, 2003, $336 \mathrm{p}$.

VIGNEAULT, Michel, La renaissance d'un sport organisé au Canada : le bockey à Montréal (1875-1917), Ph.D. (Histoire), Université Laval, 2001, 2 volumes.

VOISINE, Nive, «D’Église et d'université : Louis-Adolphe Pâquet (1859-1942) ", dans Gilles Routhier et Jean-Philippe Warren (dir.), Les iisages de la foi : figures marquantes du catholicisme québécois, Montréal, Fides, 2003, p. 123-132.

WATKINS, Meredith G., "The Cemetery and Cultural Memory: Montreal (1860-1900) », Urban History Retien/Rerue d'bistoire urbaine, 31, 1 (automne 2002), p. 52-62.

\section{Les idées, la ville et l'usine (1880-1929)}

ADAMS, Annmarie et Peter GOSSAGE, « Chez Fadette: Girlhood, Family, and Private Space in Late Nineteenth-Century Saint-Hyacinthe ", Urban History Reriew/Rerue d'bistoire urbaine, 26, 2 (mars 1998), p. 56-68. 
ANCTIL, Pierre, Saint-Laurent : la Main de Montréal, Sillery, Septentrion, 2001, 109 p.

ANCTIL, Pierre, Ira ROBINSON et Gérard BOUCHARD (dirs.), Juifs et Canadiens français dans la société québécoise. Sillery, Septentrion, 2000, $197 \mathrm{p}$.

ARMENI, Elizabeth, Menstruation Goes Public: Aspect of Women's menstrual experience in Montreal (1920-1975), M.A. (Histoire), Université McGill, 1996, 185 p.

ARMISTRONG, Elizabeth H., Le Québec et la crise de de la consiription, 1917-1918, Montréal, VLB éditeur, 1998 [1937], 293 p.

AUBRY, Jean-Christian, Pratiques religieuses et vie paroissiale en milieu urbain au XXe siècle : la paroisse Immaiulée-Conception de Montréal (19101940), M.A. (Histoire), Université de Montréal, 1998, 109 p.

AUNGER, Edmund A., "Justifying the End of Official Bilingualism: Canada's North-West Assembly and the Dual Language Question (1889-1892) ", Canadian Journal of Political Sicence/Reine canadienne de science politique, 34, 3 (2001), p. 451-486.

BASTIEN, Céline, Les syndicats internationaux et les réformes scolaires an Québec (1900-1930), M.A. (Histoire), Université de Montréal, 1997, $110 \mathrm{p}$.

BEAUDET, Marie-Andrée et Denis SAINT-JACQUES, « Lectures et critiques de la littérature française contemporaine au Québec à la fin du XIXe siècle », Études françaises, 32, 3 (1996), p. 7-20.

BEAUDREAU, Sylvie, "Déconstruire le rêve de nation : Lionel Groulx et la Révolution tranquille ", Rerue d'histoire de l'Amérique française, 56, 1 (été 2002), p. 29-61.

BÉLANGER, Damien-Claude, «La pensée ouvrière et sociale de Joseph-Papin Archambault, s.j. (1880-1966) », Bulletin du RCHTQ, 25, 2 (automne 1999), p. 13-36.

BÉLANGER, Damien-Claude, «L'abbé Lionel Groulx et la survivance franco-américaine », Francophonies d'Amérique, 13 (été 2002), p. 91-105. 
BÉLANGER, Damien-Claude, «Lionel Groulx and FrancoAmerica ", The American Retien' of Canadian Studies, 33, 3 (fall 2003), p. 373-389.

BÉLANGER, Noël, $M^{s}$ Georges Courihesne, 1880-1950, Rimouski, Archevêché de Rimouski, 2000, 223 p.

BÉLANGER, Réal, « Sir Wilfrid Laurier », Dictionnaire biographique du Canada, XIV (de 1911 à 1920), Québec, Presses de l'Université Laval, 1998, p. 664-683. http://www.biographi.ca.

BELLAVANCE, Claude et Paul-André LINTEAU, «La diffusion de l'électricité à Montréal au début du XXe siècle », dans Horacio Capel et Paul-André Linteau (dirs.), Barcelona-Montréal. Desarallo urbano comparado/Déreloppement urbain comparé, Barcelone, Publications de la Universitat de Barcelona, 1998, p. 238-258.

BERGERON, René, Encadrement clérical en contexte d'urbanisation à Shaninigan (1908-1930), M.A. (Études québécoises), Université du Québec à Trois-Rivières, 1997, 166 p.

BERNARD, Jean-Paul, "Pierre Boucher de La Bruère ", Dictionnaire biographique du Canada, XIV (de 1911 à 1920), Québec, Presses de l'Université Laval, 1998, p. 117-119.

http://www.biographi.ca.

BERTRAND, Réal, Lionel Grouli, MIontréal, Lidec, 1997, 61 p.

BIENVENUE, Louise, "Pierres grises et mauvaise conscience : essai historiographique sur le rôle de l'Église catholique dans l'assistance au Québec », Études d'histoire religieuse/Historical Studies. The Canadian Catholic Historical Association, 69 (2003), p. 9-28.

BOCK, Michel, "Le Québec a charge d'âme" : L'Action française de Montréal et les minorités françaises (1917-1928) ", Reı'ue d'histoire de l'Amérique française, 54, 3 (hiver 2001), p. 345-384. 
BOCK, Michel, "Suicide de race" ou "vocation apostolique" ? La représentation des Franco-Américains dans L'Action française de Montréal (1917-1928) ", dans Jean-Pierre Wallot (dir.), avec la collaboration de Pierre Lanthier et Hubert Watelet, Constructions identitaires et pratiques sociales: actes du colloque en hommage à Pierre Salard, tenu à l'Unicersité d'Ottawa les 4, 5, 6 octobre 2000, Ottawa, Les Presses de l'Université d'Ottawa, 2002, p. 175-200.

BOCK, Michel, Lionel Groulx, les minorités françaises et la construction de l'identité canadienne-française. Étude d'histoire intellectuelle, Ph.D. (histoire), Université d'Ottawa, 2002, ix-401 p.

BOCK, Michel, «Les Franco-Ontariens et le "réveil" de la nation : la crise du Règlement XVII dans le parcours intellectuel de Lionel Groulx », Francophonies d'Amérique, 13 (été 2002), p. 157-177.

BOILY, Frédéric, "Catholicisme et nationalisme chez Lionel Groulx », Études canadiennes/Canadian Studies, 27, 51 (2001), p. $187-$ 200.

BOILY, Frédéric, La pensée nationaliste de Lionel Grouli; Sillery, Septentrion, 2003, 229 p.

BOILY, Frédéric, «Un chevalier du catholicisme : Lionel Groulx (1878-1967) ", dans Gilles Routhier et Jean-Philippe Warren (dir.), Les iisages de la foi : figures marquantes du catholicisme québécois, Montréal, Fides, 2003, p. 161-177.

BONVILLE, Jean de, "Le "nouveau journalisme" américain et la presse québécoise à la fin du XIXe siècle ", dans Florian Sauvageau (dir.), V ariations sur l'influence culturelle américaine, Sainte-Foy, Les Presses de l'Université Laval, 1999, p. 73-100.

BOUCHARD, Gérard, «Les rapports avec la communauté juive : un test pour la nation québécoise ", dans Piere Anctil, Ira Robinson, Gérard Bouchard (dirs.), Juifs et Canadiens français dans la société québécoise. Sillery (Québec), Septentrion, 2000, p. 13-31.

BOUCHARD, Catherine, Les nations québécoises dans l'Action

Nationale : de la déiolonisation à mondialisation, [Sainte-Foy], Les Presses de l'Université Laval, 2002, 146 p 
BOUCHARD, Gérard, «Economic Inequalities in Saguenay Society, 1879-1949: A Descriptive Analysis ", The Canadian Historical Relien, 79, 4 (décembre 1998), p. 660-690.

BOUCHARD, Gérard, Les deux chanoines : contradictions et ambiralence dans la pensée de Lionel Groulx; Montréal, Boréal, 2003, 312 p.

BOUCHER, Marie-France, La construction du discours médiatique sur le cinéma au Québec (1896-1939), M.A. (Sociologie), Université Laval, 2000, 174 p.

BOUTET, Daniel, Le mourement d'opposition au monopole de l'électricité à Québec dans l'entre-deux-guerres, M.A. (Études québécoises), Université du Québec à Trois-Rivières, 1999, 113 p.

BOUVY, Emmanuelle-Minnie, Talons hauts et bas de soie : les téléphonistes de Bell Téléphone à Montréal (1920-1939), M.A. (Histoire), Université du Québec à Montréal, 2001, vi-100 p.

BRASSARD, Michèle et Jean HAMIELIN, "Aristide Filiatreault », Dictionnaire biographique du Canada, XIV (de 1911 à 1920), Québec Presses de l'Université Laval, 1998, p. 384-387.

http://www.biographi.ca.

BRASSARD, Michèle et Jean HAMELIN, «Dominique-Ceslas Gonthier ", Dictionnaire biographique du Canada, XIV (de 1911 à 1920), Québec, Presses de l'Université Laval, 1998, p. 453-456. http://www.biographi.ca.

BRAZEAU, Diane, L'organisation du loisir urbain et le rapport aux. ialeurs traditionnelles: Québec au XXe siècle, M.A. (Histoire), Université Laval, 1996, $101 \mathrm{p}$.

BRINDAMIOUR, Michel (dir.), Autour de Duns Siot. Polémique, [s.1.], Édition du Beffroi, 1989, 84 p.

BRISSON, Frédéric, «La librairie Déom au début du XXe siècle : l'édification d'un réseau d'influence par le commerce du livre ", dans Pierre Rajotte (dir.), Lieux et réseaux de sociabilité littéraire au Québec; Québec, Éditions Nota bene, 2001, p. 189-226. 
BROUSSEAU, Francine, et al. (dirs.), Litraison spéciale : l'héritage postal canadien, Montréal/Hull, Boréal/Musée canadien des civilisations/Musée canadien de la poste, 2000, 151 p.

BROWN, William, The Montreal Maroons: The Forgotten Stanley Cup Champions, Montréal, Véhicule Press, 1999, 160 p.

BRUNET, Manon et Pierre LANTHIER (dirs.), L'inscription sociale de l'intellectuel, Sainte-Foy/Paris, Les Presses de l'Université Laval/ L'Harmattan, 2000, 382 p.

CELLARD, Karine, Discours iritique, discours polémique : littérature et nationalisme dans le journal Les Débats (1900), M.A. (Études françaises), Université de Montréal, 2001, 135p.

CHAMBERLAND, Gilles, Chemins de l'avenir $d u$ Chanoine Lionel Grouk; M.A. (Études littéraires), Université du Québec à TroisRivières, 1987, 174 p.

CHARPENTIER, Marc, Broadway North: Musical Theatre in Montreal in the 1920s, Ph.D. (Histoire), Université McGill, 1999, 243 p.

CHARTIER, Daniel, «Hector Fabre et le Paris-Canada au cœur de la rencontre culturelle France-Québec de la fin du XIXe siècle", Études françaises, 32, 3 (automne 1996), p. 51-60.

COHEN, Yolande, «La question confessionnelle dans l'obtention du suffrage féminin au Canada ", Rerue internationale de sociologiel International Reicen of Sociology, 8, 2 (1998), p. 289-297.

COMEAU, Robert, "L'opposition à la conscription au Québec ", dans Roch Legault et Jean Lamarre (dirs.), La Première Guerre mondiale et le Canada: contributions sociomilitaires québécoises, Montréal, Méridien, 1999, p. 91-109.

COMIPAGNON, Antoine, "Brunetière au Québec ", Études françaises, 32, 3 (automne 1996), p. 115-128.

COOK, Ramsay, "Albert Laurendeau », Dictionnaire biographique du Canada, XIV (de 1911 à 1920), Québec, Presses de l'Université Laval, 1998, p. 662-664. http://www.biographi.ca. 
COOK, Ramsay, "Un médecin et son évêque : un incident dans l'histoire de la science et de la religion au Québec », Mens. Rer'ue d'histoire intellectuelle de l'Amérique française, 1, 2 (printemps 2000), p. 97-114.

CORNETT, Norman F., The Role of Religion in Lionel Groulv's Nationalist Thought, Ph.D. (Études religieuses), Université M IcGill, 2002, v-430 p.

CÔTÉ, Dany, « L'électrification urbaine et rurale (1891-1962), Saguenayensia, 44, 3 (2002), p. 12-18.

CÔTÉ, Luc et Jean-Guy DAIGLE, Publicité de masse et masse publicitaire : le marché québécois des années 1920 aux années 1960, Ottawa, Presses de l'Université d'Ottawa, 1999, 362 p.

COUTARD, Jérôme, Des valeurs en guerre. Presse, propagande et culture de guerre au Québec (1914-1918), Ph.D. (Histoire), Université Laval, 1999, $601 \mathrm{p}$.

COUTARD, Jérôme, «Presse, censure et propagande en 19141918 : la construction d'une culture de guerre ", Bulletin d'histoire politique, 8, 2-3 (printemps-hiver 2000), p. 150-171.

CROS, Laurence, "Le Canada et la peur de l'annexion américaine à l'époque victorienne, à travers les dessins politiques canadiens ", International Journal of Canadian Studies/Rerue internationale d'études canadiennes, 23 (printemps 2001), p. 157-188.

CROTEAU, Jean-Philippe, «L'héritage de l'institut du Baron Hirsch : entre la commission protestante et l'école Peretz (18901920) ", Bulletin du Regroupement des chercheurs-chercheuses en histoire des travailleurs et traiailleuses du Québec, 28, 1 (printemps 2002), p. 55-65.

DAGENAIS, Michèle, " Political Dimensions to Leisure and Culture Activities in Canadian Cities, 1880-1940», Urban History Reizen/ Relue d'histoire urbaine, 26, 1 (mai 1999), p. 55-70.

DAGENAIS, Michèle, «Entre tradition et modernité : espaces et temps de loisirs à Montréal et à Toronto au XXe siècle ", The Canadian Historical Retien', 82, 2 (juin 2001), p. 307-330. 
DAGENAIS, Michèle, «Inscrire le pouvoir municipal dans l'espace urbain : la formation du réseau des parcs à Montréal et à Toronto (1880-1940)", The Canadian Geographer/Le Géographe canadien, 46, 4 (hiver 2002), p. 347-364.

DAVID, Charles-Philippe et Béatrice PASCUAL, "Précurseur de la sécurité humaine : le sénateur Raoul Dandurand ", Études internationales, 31, 4 (décembre 2001), p. 649-674.

DEMERS, Michel, "Vive la Canadienne!" : les représentations de l'identité féminine dans le défilé de la Saint-Jean-Baptiste à Montréal (19241961), M.A. (Histoire), Université de Sherbrooke, 2001, vi-150 p.

DIONNE, René, Le Droit, journal culturel des Franio-Ontariens (1913-1978), Ottawa, Éditions du Vermillon, coll. « Essais et recherches ", 2002, $470 \mathrm{p}$.

DORION, Marie-Josée, Le processus d'électrification rurale $d u$ Centre du Québec, rive sud du flemre (1920-1963), M.A. (Études québécoises), Université du Québec à Trois-Rivières, 1997, 171 p.

DORION, Marie-Josée, "L'électrification du monde rural québécois ", Rel'ue d'histoire de l'Amérique française, 54, 1 (été 2000), p. 3-37.

DUBOIS, Patrick, Marius Barbeau : folklore et nationalisme, M.A. (Histoire), Université du Québec à Montréal, 2003, iv-121 p.

DUGUAY, Rodolphe, Journal (1907-1927), texte intégral, présenté et annoté par Jean-Guy Dagenais, Montréal, Éditions Varia, 2002, $752 \mathrm{p}$.

DUMONT, Micheline et Louise TOUPIN (dirs.), La pensée féministe au Québec : anthologie (1900-1985), Montréal, Éditions du Remue-ménage, 2003, 750 p.

DUPUIS, Jean-Claude, «Le Canada français et les États-Unis », Cahiers de Jeune Nation, 11 (juin 1995), p. 3-13.

EAST, Jocelyn, Les dynamismes organisationnels de l'institutionnalisation du sport au Québec (1900-1967), Ph.D. (Histoire), Université Laval, 2002, xiii-408 p. 
EWEN, Geoffrey, The International Unions and Workers' Reiolt in Quebec (1914-1925), Ph.D. (Histoire), York University, 1998, 502 p.

EWEN, Geoffrey, " Montréal Catholic School Teachers, International Unions, and Archbishop Bruchési: The Association de bienêtre des instituteurs et institutrices de Montréal (1919-1920) ", Historical Studies in Education/Rerue d'histoire de l'éducation, 12, 1-2 (printemps-automne 2000), p. 54-72.

FABRE, Gérard, «Le comparatisme d'André Siegfried », Recherches sociographiques, 43, 1 (2002), p. 111-131.

FAY, Terence J., "The Canadian Messenger of the Sacred Heart (1905-1927). Window on Ultramontane Spirituality », Etudes d'histoire religieuse/ Historical Studies. The Canadian Catholic Historical Association, 64 (1998), p. 9-26.

FECTEAU, Jean-Marie, «La dynamique sociale du catholicisme québécois au XIXe siècle : éléments pour une réflexion sur les frontières et les conditions historiques de possibilité du "social" ", Histoire sociale/Social History, 35, 70 (novembre 2002), p. 495-515.

FERRETTI, Lucia et Karl-Xavier THOMLAS, « Montée de l'Étatprovidence et transformations du soutien laïc aux œuvres d'assistance catholiques : Trois-Rivières (1921-1965)", Annales de Bretagne et des Pays de l'Ouest, 110, 4 (2003), p. 171-184.

FOISY-GEOFFROY, Dominique, Esdras Minitlle, Le catholicisme social et le nationalisme économique (1923-1939), M.A. (Histoire), Université de Montréal, 2001, x-216 p.

FOISY-GEOFFROY, Dominique, «Esdras Minville et le nationalisme économique (1923-1939) », Mens. Reine d'bistoire intellectuelle de l'Amérique française, 1, 1 (2000), p. 51-68.

FOISY-GEOFFROY, Dominique, "L'historie dans l'œuvre d'Esdras Minville ", Cahiers d'histoire, 20, 1 (2000), p. 22-44.

FOISY-GEOFFROY, Dominique, "Documents. "La vocation de la race française en Amérique" de Monseigneur Louis-Adolphe Pâquet ", Mens. Rer'ue d'histoire intellectuelle de l'Amérique française, 3, 1 (automne 2002), p. 61-95. 
FOISY, Paul, «T.-D. Bouchard : un acteur important de la culture sportive maskoutaine ", Bulletin d'bistoire politique, 11, 2 (hiver 2003), p. $53-61$.

FOULON, Arnaud, Georges Pelletier et limmigration : la pensée d'un journaliste nationaliste (1910-1939), M.A. (Histoire), Université de Montréal, 2000, 177 p.

FOURNIER, Pierre, De lutte en turlutte. Une bistoire du moutement outrier québécois à traiers ses chansons, Sillery, Septentrion, 1998, 200 p.

FRÉCHETTE, Louis, Lettres à l'abbé Baillargé : à propos d'éducation, Saint-Laurent, Bibliothèque québécoise, 2003, 266 p.

GAFFIELD, Chad, «Linearity, Nonlinearity, and the Competing Constructions of Social Hierarchy in Early Twentieth-Century Canada: The Question of Language in 1901 ", Historical Methods, 33,4 (2000), p. 255-260.

GAGNÉ, Gilles (dir.), L'antilibéralisme au Québec an XXe siècle, Québec, Éditions Nota bene, coll. «Société », 2003, 345 p.

GAGNÉ, Gilles et Jean-Philippe WARREN, Sociologie et raleurs: quatorie penseurs québéciois du XXe siècle, Montréal, Presses de l'Université de Montréal, coll. «PUM-corpus », 2003, 396 p.

GAGNON, Nicole, «Sur le présumé maurrassisme de Lionel Groulx ", Les Cabiers d'histoire du Québec au XXe siècle, 8 (automne 1997), p. 88-93.

GAGNON, Nicole, "Catherine Pomeyrols. Les intellectuels québécois: formation et engagements (1919-1939) », Recheribes sociographiques, 38, 2 (1997), p. 377-382.

GALLICHAN, Gilles, «De Québec à San Francisco : N.-E. Dionne en Californie en 1915 », Les Cabiers des Dix, 53 (1999), p. 79-115.

GAUDREAU, Guy et Nicheline TREMBLAY, «Harry Bernard (1898-1979) : érudit et homme de lettres ", Mens. Rerue d'bistoire intellectuelle de l'Amérique française, 2, 1 (automne 2001), p. 35-66. 
GAUVREAU, Danielle, "Rats des villes et rats des champs : populations urbaines et populations rurales du Québec au recensement de 1901 », Cahiers québécois de démographie, 30, 2 (automne 2001), p. 171-190.

GIGUÈRE, Richard, “"Ces restes d'Inquisition...” Littérature, édition et censure dans les correspondances d'écrivains de l'entredeux-guerres au Québec ", I'oix et images, 68, 2 (hiver 1998), p. 248-265.

GIRARD, Camil, Canada: A Country Divided: The Times of London and Canada (1908-1922), Chicoutimi, Groupe de recherche et d'intervention régionales, Université du Québec à Chicoutimi, 2001, 242 p.

GIRARD, Denise, «Les débuts dans la jeunesse bourgeoise montréalaise (1920-1940) ", dans Gérard Bouchard et Martine Segalen (dirs.), Une langue, deux cultures : rites et symboles en France et au Québec, Sainte-Foy/Paris, Les Presses de l'Université Laval/ Éditions La Découverte, 1997, p. 249-263.

GOSSELIN, Line, Les journalistes québécoises (1880-1930), Ph.D. (Histoire), Université du Québec à Montréal, 1994, 174 p.

GREEN, Alan et Mary MLACKINNON, "The Slow Assimilation of British Immigrants in Canada: Evidence from Montreal and Toronto, 1901 », Explorations in Economic History, 38, 3 (2001), p. 315-338.

GROULX, Lionel, Correspondance (1894-1967). L'intellectuel et l'bistorien norices (1909-1915), III, Édition critique par Gisèle Huot, Juliette Lalonde-Rémillard, Pierre Trépanier, Montréal, Fides, 2003, 1064 p.

GROULX, Patrice, "Benjamin Sulte, père de la commémoration ", Journal of the Canadian Historical Association/Rerue de la Société bistorique du Canada, 12 (2001), p. 49-72.

HAMEL, Thérèse, "Jean-Baptiste Cloutier ", Dictionnaire biographique du Canada, XIV (de 1911 à 1920), Québec, Presses de l'Université Laval, 1998, p. 242-244. http://www.biographi.ca. 
HARVEY, Fernand, "La politique culturelle d'Athanase David (1919-1936) », Les Cabiers des Dix, 57 (2003), p. 31-83.

HARVEY, Janice, "Dealing with "the Destitute and the Wretched": The Protestant House of Industry aud Refuge in Nineteenth-Century Montreal ", Journal of the Canadian Historical Association/Rerue de la Société historique du Canada, 12 (2001), p. 7394.

HARVEY, Pierre, Histoire de l'École des hautes études commerciales de Montréal (1926-1970), tome 2, Montréal, Québec Amérique, 2002, $448 \mathrm{p}$.

HAUSER, Claude et Yvan LAMONDE (dir.), Regards iroisés entre le Jura, la Suisse romande et le Québec; Sainte-Foy/Porrentruy (Suisse), Presses de l'Université Laval/Office du patrimoine et de la culture de la République et Canton du Jura, 2002, 352 p.

HÉBERT, Karine, Une organisation maternaliste au Québec, la Fédération nationale Saint-Jean-Baptiste (1900-1940), M.A. (Histoire), Université de Montréal, 1997, $118 \mathrm{p}$.

HÉBERT, Karine, «Une organisation maternaliste au Québec : la Fédération nationale Saint-Jean-Baptiste et la bataille pour le vote des femmes ", Rel'ue d'histoire de l'Amérique française, 52, 3 (hiver 1999), p. 315-344.

HÉBERT, Karine, La construction d'une identité étudiante montréalaise (1895-1960), Ph.D. (Histoire), Université du Québec à Montréal, 2002, ix-359 p.

HÉBERT, Pierre (dir.), «La censure, 1920-1960 », numéro spécial de Voiv et images, 23, 2 (hiver 1998), p. 217-325.

HÉBERT, Pierre, " "Où est l'univers concentrationnaire ?” : Le Deioir et les paradigmes de la censure (1920-1960)", Voix et images, 23, 2 (hiver 1998), p. 229-247.

HÉBERT, Pierre, « Une censure totale? L’Église québécoise et la nationalisation de l'imaginaire littéraire (1920-1929) », Études d'bistoire religieuse/ Historical Studies. The Canadian Catholic Historical Association, 67 (2001), p. 293-300. 
HERON, Craig et Steve PENFOLD, "The Craftmen's Spectacle: Labour Day Parades in Canada, The Early Years ", Histoire sociale/ Social History, 29, 58 (novembre 1996), p. 357-389.

HOUDE, Patricia, Lionel Groulx: un traditionaliste à la rencontre de l'Europe (1921-1922), M.A. (Histoire), Université de Montréal, 2000, vi-119 p.

HUDON, Christine, "La sociabilité religieuse à l'ère du vapeur et du rail ", Journal of the Canadian Historical Association/Rerue de la Société historique du Canada, 10 (2000), p.129-148.

JONES, Preston, « Protestants, Catholics, and the Bible in LateNineteenth-Century Quebec », Fides et Historia, 33, 2 (2001), p. 3138 .

JUTEAU, Danielle et Nicole LAURIN, Un métier et une rocation. Le trarail des religieuses au Québec de 1901 à 1971, Montréal, Les Presses de l'Université de Montréal, 1997, 194 p.

LACOMBE, Alain, «Les intellectuels québécois dans leurs mémoires au XXe siècle, de Lionel Groulx à Gérard Pelletier », dans Manon Brunet et Pierre Lanthier (dirs.), L'inscription sociale de l'intellectuel, Sainte-Foy/Paris, Les Presses de l'Université Laval / L'Harmattan, 2000, p. 275-288.

LACOMIBE, Danyèle, Marie Gérin-Lajoie's Hidden Crucifixes: Social Catholicism. Feminism, and Québec Modernity (1910-1930), M.A. (Histoire), University of Alberta, 1998, 121 p.

LACOMBE, Sylvie, "Entre l'autorité pontificale et la liberté nationale : l'anti-impérialisme britannique d'Henri Bourassa ", dans Robert Comeau et Luc Desrochers (dirs.), Le Devoir : un journal indépendant, 1910-1995, Sainte-Foy, Les Presses de l'Université du Québec, 1996, p. 273-281.

LACONIBE, Sylvie, La rencontre de deux peuples élus : comparaison des ambitions nationale et impériale au Canada entre 1896 et 1920, SainteFoy, Les Presses de l'Université Laval, coll. « Sociologie contemporaine », 2002, $291 \mathrm{p}$. 
LACOMBBE, Sylvie, "Un prophète ultramontain : Henri Bourassa ", dans Gilles Routhier et Jean-Philippe Warren (dir.), Les lisages de la foi : figures marquantes du catholicisme québécois, SaintLaurent, Fides, 2003, p. 133-145.

LAFLAMIME, Valérie, "Familles et modes de résidence en milieu urbain québécois au début du XXe siècle : l'exemple de la ville de Québec (1901)", Cahiers québéiois de démographie, 30, 2 (automne 2001), p. 261-288.

LAGRÉE, Michel, « De Veuillot à Tardivel, ou les ambiguités de la haine de la modernité ", Études d'bistoire religieuse/Historical Studies. The Canadian Catholic Historical Association, 67 (2001), p. 251-259.

LAMONDE, Yvan, "L'Affaire Dreyfus et les conditions d'émergence de l'intellectuel vues des Amériques ", dans Marie-Christine Granjon et Michel 'Trebitsch (dirs.), Pour une histoire comparée des intellectuels, Paris, Éditions Complexe, 1998, p. 111-123.

LAMONDE, Ivan, "L'époque des francs-tireurs : les intellectuels au Québec, 1900-1930», dans Manon Brunet et Pierre Lanthier (dirs.), L'inscription sociale de l'intellectuel, Sainte-Foy/Paris, Les Presses de l'Université Laval/L'Harmattan, 2000, p. 189-211.

LAMONDE, Yvan, « Un aspect inconnu du débat autour de la bibliothèque publique de Montréal : la Montreal Free Library (1889-) ", Les Cahiers des Dix; 57 (2003), p. 263-271.

LAMOUREUX, Diane, "Une opposition farouche au suffrage féminin (1913-1940) ", dans Robert Lahaise (dir.), Le Devoir, reflet du Québec an 20e siècle, LaSalle, Hurtubise HMIH, 1994, p. 331-344.

LAMIBERT, Fernando, "Clément Lockell, frère des écoles chrétiennes, éducateur, écrivain, homme de culture (1908-1984) ", dans Gilles Routhier et Jean-Philippe Warren (dir.), Les irsages de la foi : figures marquantes du catholicisme québécois, Montréal, Fides, 2003, p. 147-159.

LAPLANTE, Jean de, Les parcs de Montréal: des origines à nos jours, Montréal, Éditions du Méridien, 1990, 255 p. 
LEBLANC, Katleen, Les intellectuels québécois et l'Affaire Dreyfus (1894-1940), M.A. (Études québécoises), Université du Québec à Trois-Rivières, 1998, $119 \mathrm{p}$.

LEBLANC, Marcel, «Les premières automobiles à Roberval, Saguenayensia, 44, 4 (2002) p. 18-21.

LEMIEUX, Raymond, "Le sourire du martyr : Gérard Raymond (1912-1932)", dans Gilles Routhier et Jean-Philippe Warren (dir.), Les visages de la foi : figures marquantes du catholicisme québécois, Montréal, Fides, 2003, p. 49-66.

LEROUX, Éric, Gustaie Franiq. Figure marquante du syndicalisme et précurseur de la FTQ, Montréal, VLB éditeur, 2001, 371 p.

LEROUX, Éric, La pensée de Gustaie Franiq, syndicaliste et réformateur social. Textes ihoisis (1905-1948), Montréal, Collection du RCHTQ, 2001, 166 p.

LONGSTAFF, Alison, Vie intellectuelle et libre pensée au tournant du XXe siècle : le cas de Ludger Larose, M.A. (Études québécoises), Université du Québec à Trois-Rivières, 1999, 230 p.

LUNEAU, Marie-Pier, Lionel Grouh : le mythe du berger, M Iontréal, Leméac, 2003, 226 p.

MAHÉ, Yvette T.M., "Bilingual School District Trustees and Cultural Transmission: The Alberta Experience (1892-1939)", Historial Studies in Education/Re'ue d'histoire de l'éducation, 9, 1 (printemps 1997), p. 65-82.

MAILHOT, Laurent, " Jules Foumier ", Dictionnaire biographique du Canada, XIV (de 1911 à 1920), Québec, Presses de l'Université Laval, 1998, p. 406-407. http://www.biographi.ca.

MLARCIL, Jeffrey, "Les nôtres" : Franco-Américains, Canadiens français hors Québec et Acadiens dans la grande presse montréalaise de langue française (1905-1906), M.A. (Histoire), Université d'Ottawa, 1998, $158 \mathrm{p}$. 
MLARCOT'TE, Gilles, «Pauvreté d'Hector de Saint-Denys Garneau (1912-1943) », dans Gilles Routhier et Jean-Philippe Warren (dir.), Les iisages de la foi : figures marquantes du catholicisme québéiois, Montréal, Fides, 2003, p. 107-120.

MLARQUIS, Dominique, La presse catholique au Québec (1910-1940), Ph.D. (Histoire), Université du Québec à Montréal, 1998, 435 p.

MLARQUIS, Dominique, "Un nouveau combat pour l'Église : la presse catholique d'information (1907-1940)», Études d'bistoire religieuse/Historical Studies. The Canadian Catholic Historical Association, 68 (2002), p. 73-88.

MLARTEL, Marcel, «Le Québec et les groupes minoritaires francophones : analyse des actions du réseau institutionnel et de l'État québécois, de la fin du XIXe siècle à 1969 ", dans Pour un renforcement de la solidarité entre franiophones au Canada : réflexions théoriques et analyses historique, juridique et sociopolitique, Québec, Conseil de la langue française, 1995, p. [119]-151.

MLARTIN, Annie, L'imprimerie l'Action sociale Ltée : l'Église catholique dans l'arène de l'édition québécoise (1907-1939), M.A. (Histoire), Université du Québec à Montréal, 2003, vi-149p.

MLARTIN, Jean-Clément et Catherine POMEYROLS, « Les clercs et l'Église du Québec et de la Vendéee face à l'Histoire, fin XIXe début XXe siècles ", dans Michel Lagrée (dir.), Chocs et ruptures en histoire religieuse. Fin XI IIIL-XIXe siècles, Rennes, Presses universitaires de Rennes, 1998, p. 191-208.

MATTHIEU, Jocelyne, "L'éducation familiale et la valorisation du quotidien des femmes au XXe siècle ", Les Cabiers des Dix; 57 (2003), p. 119-150.

McGOWAN, Mark G., "What Did Michael Power Really Want ? Questions Regarding the Origins of Catholic Separate Schools in Canada West ", Études d'bistoire religieuse / Historical Studies. Canadian Catholic Historical Association, 68 (2002), p. 85-104.

MEDRESH, Israël, Le Montréal juif entre les deux guerres, traduction de Pierre Anctil, Sillery, Septentrion, 2001, 242 p. 
MÉTHOT, Mélanie, "Jules Helbronner (1844-1921) : père de la conscience ouvrière montréalaise et intellectuel engagé ", Mens. Rerue d'bistoire intellectuelle de l'Amérique française, 2, 1 (automne 2001), p. 67-103.

MÉTHOT, Mélanie, «Herbert Brown Ames: Political Reformer and Enforcer ", Urban Historical Retiew/Rerue d'bistoire urbaine, 31, 2 (printemps 2003), p. 18-31.

MILLETTE, Renée-Marthe, Clarence A. Gagnon. Un cas de résistance à la modernité, M.A. (Histoire de l'art), Université de Montréal, 1995, 117 [54] p.

MIORIN, Rosaire, "Histoire de l'Action nationale », L'Aition nationale, 92, 1 (janvier 2002), p. 17-31.

MONTMINY, Jean-Paul, "L'entrepreneur d'œuvres dominicain : Georges-Henri Lévesque, o.p. (1903-2000) », dans Gilles Routhier et Jean-Philippe Warren (dir.), Les visages de la foi : figures marquantes du catholicisme québécois, Montréal, Fides, 2003, p. 359-371.

MONTREUIL, Sophie, " (Se) lire et (se) dire : Joséphine Marchand-Dandurand et la lecture (1879-1886), dans Yvan Lamonde et Sophie Montreuil (dirs), Lire au Québec au XIXe siècle, SaintLaurent, Fides, 2003, p. 123-150.

MUNRO, Kenneth, "Canada as Reflected in Her Participation in the Coronation of Her Monarchs in the Twentieth Century ", Journal of Historical Sociology, 14,1 (2001), p. 21-46.

NACHBAUER, Martine, Analyse du discours polémique sur la lecture dans la rerue pédagogique québécoise L'enseignement primaire (18811956), Ph.D. (Éducation), Université du Québec à Montréal, 2000, $274 \mathrm{p}$.

NADEAU, Jean-François, Robert Rumilly en son histoire, Ph.D. (Histoire), Université du Québec à Montréal, 2003, ix-539 p.

NELLES, H.V., "Historical Pageantry and the Fusion des Races at the "Tercentenary of Quebec", 1908 ", Histoire sociale/Social History, 29, 58 (novembre 1996), p. 391-416. 
NELLES, H.V., The Art of Nation Building: Pageantry and Spectacle at Quebei's Terientenary, Toronto, University of Toronto Press, 1999, $408 \mathrm{p}$.

NELLES, H.V., L'bistoire spectacle : le cas du tricentenaire de Québec, traduit de l'anglais par Hélène Paré, Montréal, Boréal, 2003, 428 p.

NOOTENS, Thierry, Fous, prodigues et ilrognes : internormativité et dériance au Québec au 19 e siècle, Ph.D. (Histoire), Université du Québec à Montréal, 2003, ix-403 p.

NOPPEN, Luc et Lucie K. Morisset, «La Maison québécoise : Construction et déconstruction d'un emblème ", Joumal of Canadian Art History / Annales d'histoire de l'art canadien, 22, 1-2 (2001), p. 2667.

NORCLIFFE, Glen, Ride to Modernity: The Bicycle in Canada, 18961900, Toronto, University of Toronto Press, 2001, 248 p.

OLSON, Sherry and Patricia THORNTON, "The Challenge of the Irish Catholic Community in Nineteenth-Century Montreal ", Histoire sociale/Social History, 35, 70 (2002), p. 331-362.

PEARSON, Robert H., Montreal's Delorimier Downs Baseball Stadium as Business and Centre of Mass Culture (1928-1960), M.A. (Histoire), Queen's University, 1998, 140 p.

PELLETIER, Louis-Raphaël, Nationalisme et libéralisme dans la pensée de Jules Fournier (1904-1917), M.A. (Histoire), Université de Montréal, 1999, 138 p.

PELLETIER-BAILLARGEON, Hélène, Olicar Asselin et son temps : le iolontaire, tome 2, M Contréal, Fides, 2001, 321 p.

PERIN, Roberto, "Adélard Langevin ", Dictionnaire biographique du Canada, XIV (de 1911 à 1920), Québec, Presses de l'Université Laval, 1998, p. 650-655. http://www.biographi.ca.

PICHÉ, Sébastien, Histoire de la spécialisation médicale au Québec : Le cas de la naissance de l'Hématologie à l'hôpital Notre-Dame de Montréal (19201960), M.A. (Histoire), Université du Québec à Montréal, 1999, $122 \mathrm{p}$. 
PILON, Simone, Constitution du corpus des écrits des femmes dans la presse canadienne-française entre 1883 et 1893 et analyse de l'usage des pseudonymes, Ph.D. (Littérature), Université Laval, 1999, 280 p.

PLANTE, Charlotte, "Militante et croyante. Une figure de proue du syndicalisme enseignant au Québec : Laure Gaudreault (18901975) », dans Gilles Routhier et Jean-Philippe Warren (dir.), Les izsages de la foi : figures marquantes du catholicisme québécois, SaintLaurent, Fides, 2003, p. 329-348.

PLEAU, Jean-Christian, "Polémique sur un "mauvais livre" : L'appel de la race de Lionel Groulx ", Voix et Images, 28, 2 (83) (2003), p. 138-159.

POIRIER, Claude-Anne, La dictée au Québec entre 1898 et 1923 : une leçon de langue ou une leçon de morale? M.A. (Éducation), Université du Québec à Montréal, 1999, 239 p.

POITRAS, Claire, La cité au bout du fil: le téléphone à Montréal de 1879 à 1930, Montréal, Les Presses de l'Université de Montréal, 2000, 323 p.

POMEYROLS, Catherine, "Les intellectuels nationalistes québécois et la condamnation de l'Action française ", I ingtième siècle. Recue d'histoire, 73, (janvier-mars 2002), p. 83-98.

PROULX, Serge, "La "poésie” de Saint-Denys Garneau et la crise identitaire au Canada français », Liberté, 45, 1, 259 (février 2003), p. 89-102.

PRUD'HOMIME, Annie-Claude, "Voyage autour d'une bibliothèque : la littérature dans les catalogues de bibliothèques personnelles d'écrivains (1880-1910) », dans Yvan Lamonde et Sophie Montreuil (dirs), Lire au Québec au XIXe siècle, Saint-Laurent, Fides, 2003, p. 159-200.

RAJOTTE, Pierre, «La représentation de l'autre dans les récits de voyage en Terre Sainte à la fin du XIXe siècle », Études françaises, 32, 3 (1996), p. 95-113. 
RAMIREZ, Bruno, "Canada in the United States: Perpectives on $M$ ligration and Continental History ", Journal of American Ethnic History, 20, 3 (2001), p. 50-70.

REID, Dennis (dir.), Krieghoff: Images of Canada, Vancouver, Douglas \& McIntyre, 1999, 360 p.

Traduction française : Krieghoff : images $d u$ Canada, Saint-Laurent, Trécarré, 1999, 323 p.

ROBERT, Lucie, " L'art de transformer une défaite en victoire : la représentation des Rébellions dans le théâtre de Louis Fréchette », Bulletin d'histoire politique, 12, 1 (automne 2003), p. 16-27.

ROBIN, Martin, Le spectre de la droite. Histoire des politiques nativistes et fascistes au Canada entre 1920 et 1940, Montréal-Paris, Balzac-Le Griot éditeur, 1998, 306 p.

Traduction de Shades of Right: Nativist and Fascist Politics in Canada, 1920-1940, Toronto, University of Toronto Press, 1992, 372 p.

RODRIGUE, Martine, "Les Franco-Américains à Montréal en 1901 : un regard sur le retour au pays ", Francophonies d'Amérique, 9 (1999), p. 107-115.

ROY, Fernand, "L'histoire dans les romans de Laure Conan. Lecture sémiotique de l'idéologie de la langue gardienne de la foi ", I oix et images, 25, 2 (hiver 2000), p. 328-348.

ROY, Fernande et Jean de BONVILLE, "La recherche sur l'histoire de la presse québécoise : bilan et perspectives », Recherihes sociographiques, 41, 1 (janvier-avril 2000), p. 15-51.

ROY, Fernande, "Les intellectuels canadiens-français du siècle dernier : les journalistes », dans Manon Brunet et Pierre Lanthier (dirs.), L'inscription sociale de l'intellectuel, Sainte-Foy/Paris, Les Presses de l'Université Laval/L'Harmattan, 2000, p. 341-350.

ROUILLARD, Jacques, "La défense de l'autonomie provinciale en matière de politiques sociales : le cas de la Confédération des travailleurs catholiques du Canada (1919-1960) ", Cahiers d'bistoire, 17, 1-2 (printemps-automne 1997), p. 95-108. 
ROYER, Julie, La subiersion du discours clérical dans trois romans carnaralesques du Québec; M.A. (Études littéraires), Université du Québec à Montréal, 1999, 151 p.

RUDIN, Ronald, " M Iarching and Memory in Early TwentiethCentury Quebec: La Fête-Dieu, la Saint-Jean-Baptiste, and the Monument Laval ", Journal of the Canadian Historical Association/ Rerue de la Société historique du Canada, 10 (2000), p. 209-236.

RUDY, Jarrett, "Unmaking Manly Smokes: Religion, the State, and the First Anti-Smoking Campaigns in Montreal (1892-1914)", Journal of the Canadian Historical Association/Rerue de la Société historique du Canada, 12 (2001), p. 95-114.

RYAN, Pascale, «Des intellectuels en Europe et en Amérique : un état de la question ", Mens. Rerue d'histoire intellectuelle de l'Amérique française, 4, 1 (automne 2003), p. 9-37.

SAINT-YVES, Gabrielle, "L'idéologie à travers les questions de langue : riposte de Firmin Paris à la chronique de langue de Louis Fréchette ", Globe. Relue internationale d'études québécoises, 6, 2 (2003), p. 123-146.

SALVATORE, Filippo, Fascism and the Italians of Montreal: an Oral History (1922-1945), Toronto, Guernica, 1998, 96 p.

SARAULT, Julie, La mise en place des structures de la recherche universitaire : la Faculté des sciences de l'Université de Montréal (1920-1945), M.A. (Histoire), Université du Québec à Montréal, 2000, 135 p. SAVARD, Pierre, "Relations avec le Québec », dans Cornelius J. Jaenen (dir.), Les Franio-Ontariens, Ottawa, Les Presses de l'Université d'Ottawa, 1993, p. 231-263.

SAVOIE, Chantal, «Des salons aux annales : les réseaux et associations des femmes de lettres à Montréal au tournant du XXe siècle ", Voix et images, 27, 2 (hiver 2002), p. 238-253. 
SCHMIIDT, Sarah, "Private" Acts in "Public" Spaces: Parks in Turn-of-the-Century Montreal ", dans Power, Place and Identity: Historical Studies of Social and Legal Regulation in Quebec; actes d'un colloque du Groupe sur l'bistoire de Montréal (1996), Montréal, The Group, 1998, p. 129-149.

STAGNI, Pellegrino Francesco, The I iew from Rome: Arcbbishop Stagni's 1915 Reports on the Ontario Bilingual Schools Question, translated by John Zucchi, M Iontréal et Kingston, McGill-Queen's University Press, 2002, 134 p.

TAJUELO, Telesforo, Censure et société : un siècle d'interdit cinématographique au Québec, Ph.D. (Études cinématographiques), Paris III, 1998, 2 tomes et annexes.

TRÉPANIER, Esther, Peinture et modernité au Québec; 1919-1939, Québec, Éditions Nota bene, 1998, 395 p.

TRÉPANIER, Pierre, "Lionel Groulx, historien », Les Cahiers des Dix, 47 (1992), p. 247-277.

TRÉPANIER, Pierre, «Un projet d'enquête sur la jeunesse universitaire (1913)", Les Cahiers des Dix; 54 (2000), p.137-169.

TRÉPANIER, Pierre, "L'étudiant idéal vers 1913 », Les Cahiers des Dix; 55 (2001), p.117-148.

TULCHINSKY, Gerald, Branching Out: The Transformation of the Canadian Jenish Community, North York (Ont.), Stoddart Publishing, 1998, 416 p.

TURGEON, Laurier, Jocelyn LÉTOURNEAU et Khadiyatoulah FALL (dirs.), Les espaces de l'identité, Sainte-Foy, Les Presses de l'Université Laval, 1997, 324 p.

VALCOUR, Pierre et François BEAUDIN, dir., La liberté vient aussi de Dieu... : témoignages en l'honneur de Georges-Henri Léresque, o.p. (1903-2000), [Sainte-Foy], Presses de l'Université Laval, 2002, 328 p.

VEILLEUX, Denis, La motorisation, ou, "La rangon du progrès »: tramways, véhicules-moteurs et circulation (Montréal, 1900-1930), Ph.D. (Histoire), Université MIcGill, 1998, 440 p. 
VOISINE, Nive, "Louis Gonnet ", Dictionnaire biographique du Canada, XIV (de 1911 à 1920), Québec, Presses de l'Université Laval, 1998, p. 451-453. http://www.biographi.ca.

WARREN, Jean-Philippe, "La découverte de la "question sociale". Sociologie et mouvements d'action jeunesse canadiensfrançais ", Relue d'bistoire de l'Amérique française, 55, 4 (printemps 2002), p. 539-572.

WARREN, Jean-Philippe, L'engagement sociologique : la tradition sociologique du Québec francophone (1886-1955), Montréal, Boréal, 2003, 448 p.

WARREN, Jean-Philippe, "Inquiétudes et espoirs au Québec en 1900 », Argument, 6, 1 (automne 2003-hiver 2004), p. 124-132.

WILLIS, John, "L'importance sociale du bureau de poste en milieu rural au Canada (1880-1945)", Histoire sociale/Social History, 30, 59 (mai 1997), p. 143-168.

\section{Une crise intellectuelle (1929-1945)}

ALMIEIDA, Mike, L'office protincial des recherches scientifiques et le déreloppement de la sicience au Québec, 1937-1960, M.A. (Histoire), Université du Québec à Montréal, 2002, 76 p.

AMYOT, Éric, La bataille pour le Québec: Vichy. La France libre et les Canadiens français (1940-1945), Ph.D. (Histoire), Université MIcGill, 1998, 452 p.

AMYOT, Éric, Le Québec entre Pétain et de Gaulle : Vichy, la France libre et les Canadiens français, 1940-1945, Saint-Laurent, Fides, 1999, $365 \mathrm{p}$.

AMYOT, Éric, «Vichy, la France libre et le Canada français : bilan historiographique », Bulletin d'bistoire politique, 7, 2 (hiver 1999), p. 917.

ANCTIL, Pierre, "Réponse à Esther Delisle sur l'antisémitisme ", dans André Champagne (dir.), Le Québec contemporain, Sillery, Septentrion, 1995, p. 55-68. 